\title{
Produtividade da cultura da abóbora em diferentes densidades de plantio no Vale do São Francisco
}

\author{
Geraldo M de Resende; Rita Mércia E Borges; Nadja Pollyanna S Gonçalves
}

Embrapa Semiárido, C. Postal 23, 56300-000 Petrolina-PE; geraldo.milanez@embrapa.br; rita.faustino@embrapa.br; nadja.goncalves@, embrapa.br

\section{RESUMO}

A abóbora é amplamente cultivada e consumida no Brasil, em especial na região Nordeste. O espaçamento entre e dentro da linha são cruciais para o desenvolvimento e produção da cultura. Com o objetivo de avaliar diferentes espaçamentos de plantio na produtividade de acessos e cultivar de abóbora, instalou-se um experimento no período de maio a setembro de 2010, no campo experimental da Embrapa Semiárido em Petrolina-PE. O delineamento experimental utilizado foi de blocos ao acaso, em esquema fatorial $5 \times 3$, compreendendo quatro acessos selecionados do Banco Ativo de Germoplasma de Abóbora (Acessos: A422, A612, A620 e A627) e a cultivar Jacarezinho como testemunha, e três espaçamentos entre plantas (4x1; 4x2 e 4x3 m), com quatro repetições. Maior produtividade foi obtida pelo acesso A612 (17,3 $\left.\mathrm{t} \mathrm{ha}^{-1}\right)$, seguido pelo acesso A422 $\left(15,2 \mathrm{tha}^{-1}\right) \mathrm{e}$, o pior desempenho foi obtido pela cultivar Jacarezinho $\left(12,2 \mathrm{tha}^{-1}\right)$, sem diferenciar dos demais acessos. Para espaçamento entre plantas observou-se que as maiores produtividades foram verificadas no menor espaçamento $(4 \mathrm{x} 1 \mathrm{~m})$ com $18,2 \mathrm{tha}^{-1}$, e menores produtividades no espaçamento de $4 \times 3 \mathrm{~m}\left(11,9 \mathrm{t} \mathrm{ha}^{-1}\right)$. O acesso A612 sobressaiu-se com maior massa fresca $\left(3,5 \mathrm{~kg}_{\text {fruto }}{ }^{-1}\right)$, não se diferenciando estatisticamente do acesso A422, que alcançou $3,2 \mathrm{~kg}$ fruto $^{-1}$. A cultivar Jacarezinho e o acesso A620, com respectivos 1,9 e $2,0 \mathrm{~kg}$ fruto $^{-1}$ obtiveram os menores valores para massa fresca do fruto, assim como maior número de frutos por planta com 4,7 e 4,6 frutos plantas $^{-1}$, respectivamente.

Palavras-chave: Cucurbita moschata, produtividade, espaçamentos, cultivares, acessos.

\begin{abstract}
Yield of pumpkin crop in different planting densities in the São Francisco Valley

Pumpkin is extensively cultivated and consumed in Brazil, in a special way in Northeast Region. The spacing between and within the line is crucial for the development and production of the crop. The experiment was carried out in Petrolina, Pernambuco state, Brazil, from May to September, 2005. We evaluated different planting spacings on yield of pumpkin accessions and one cultivar. The experimental design was complete randomized blocks in factorial scheme $5 \times 3$, consisting of five selected accessions from the Germplasm Active Bank of Pumpkin (accessions A422, A612, A620 and A627) and Jacarezinho cultivar as control, and three spacing values within planting rows $(4 \times 1 ; 4 \times 2$ and $4 \times 3 \mathrm{~m})$ with four replications. Highest yield was presented by accession A612 (17.3 $\left.\mathrm{t} \mathrm{ha}^{-1}\right)$, followed by accession A422 (15.2 $\left.\mathrm{t} \mathrm{ha}^{-1}\right)$, with worst performance from cultivar Jacarezinho $\left(12.2 \mathrm{t} \mathrm{ha}^{-1}\right)$, without differing from the other treatments. For plant spacing the highest yields were obtained in the smaller spacing $(4 \mathrm{x} 1 \mathrm{~m})$ with $18.2 \mathrm{tha}^{-1}$, and lower yield in the spacing of $4 \times 3 \mathrm{~m}\left(11.9 \mathrm{tha}^{-1}\right)$. The accession A612 showed the highest fresh fruit mass $\left(3.5 \mathrm{~kg}_{\text {fruit }}{ }^{-1}\right)$ without statistical difference from accession A422 (3.2 kg fruit $\left.{ }^{-1}\right)$. The cultivar Jacarezinho and accession A620 with respectively 1.9 and $2.0 \mathrm{~kg}_{\text {fruit }}^{-1}$ presented the lowest values of fresh fruit mass as well as the largest number of fruits per plant (4.7 and 4.6, respectively).
\end{abstract}

Keywords: Cucurbita moschata, yield, spacings, cultivars, accessions.

(Recebido para publicação em 24 de setembro de 2012; aceito em 29 de agosto de 2013) (Received on September 24, 2012; accepted on August 29, 2013)

\begin{abstract}
A família Cucurbitaceae, grupo vegetal que ocorre nas regiões tropicais do mundo, é formada por cerca de 120 gêneros que contêm mais de 800 espécies (Teppner, 2004). No Brasil, ocorrem cerca de 30 gêneros e 200 espécies. Entre as espécies de importância econômica e alimentar destacam-se as abóboras (Cucurbita moschata e Cucurbita pepo), moranga (Cucurbita maxima) e o mogango (Cucurbita pepo) (Ferreira et al., 2006). A abóbora (C. moschata) tem como centro de origem as Américas, com significativa participação na alimentação de muitos países.

Além do valor econômico e alimen-
\end{abstract}

tar, o cultivo de cucurbitáceas no Brasil, em especial as abóboras, tem grande importância social na geração de empregos diretos e indiretos, pois demanda grande quantidade de mão-de-obra, desde o cultivo até a comercialização. A pesquisa de Orçamento Familiar 2008-2009, realizada pelo IBGE (2010) concluiu que o consumo per capita de hortaliças no Brasil foi de $27,08 \mathrm{~kg}$, sendo a participação da abóbora de $1,19 \mathrm{~kg}$, com maior consumo no Nordeste com 1,24 $\mathrm{kg}$, e maior expressão no Piauí, com $2,62 \mathrm{~kg}$.

O cultivo de abóboras (Curcubita moschata) ocorre normalmente em pequenas propriedades rurais e em cultivos comerciais; seu uso na alimentação animal é comum, devido à produtividade das plantas e à durabilidade dos frutos (Heiden et al., 2007). Na alimentação humana é usada no preparo de doces em calda ou em pasta, pratos salgados, ensopados ou cozidos. Os frutos desta espécie são conhecidos como abóbora, abóbora-crioula, abóbora de pescoço, abóbora gigante, lagarteira, abóbora de vaca, abóbora menina, moranga, jerimum, abóbora de leite, maranhão, abóbora comum, entre outros (Barbieri et al., 2006; Heiden et al., 2007).

A produção mundial de abóboras, 
em 2010, foi de 22,4 milhões de toneladas, cultivadas em área de 1,67 milhão de hectares, proporcionando uma produtividade média de 13,4 t ha-1 (FAO, 2012). No Brasil, os dados referentes à comercialização são escassos, sendo a última informação disponível em 2006, com área colhida de 88.203 ha, 384.916 $\mathrm{t}$ produzidas, que proporcionaram uma produtividade média de $4,4 \mathrm{t} \mathrm{ha}^{-1}$, com valor da produção de 1,52 milhões de reais, cultivada em mais de 127 mil estabelecimentos agropecuários (IBGE, 2012). Os estados do Nordeste representaram 24,1\% da produção nacional, sendo os maiores produtores a Bahia, Maranhão e Pernambuco. A abóbora é bastante difundida na região, onde é considerada cultura de subsistência (Carmo et al., 2011).

Entre as cucurbitáceas existentes no Nordeste brasileiro, as abóboras (Cucurbita moschata) são apreciadas por toda a população e têm grande potencial para cultivos comerciais. A ampla variabilidade da espécie tem sido estudada e conservada. Atualmente, o Banco Ativo de Germoplasma (BAG) de Cucurbitáceas da Embrapa Semiárido mantém 543 acessos conservados (Borges et al., 2011).

A densidade de plantas é um fator importante que afeta principalmente a produtividade (Latifi et al., 2012). A densidade ótima depende de diferentes fatores, tais como atributos da planta, período de crescimento, época de semeadura e manejo, fertilidade do solo, tamanho da planta, umidade disponível, radiação solar e o método de plantio (Ameri et al., 2007). O espaçamento entre e dentro da linha são cruciais para o desenvolvimento e produção de abóboras (Fanadzo et al., 2010; El -Hamed et al., 2011; Abdel-Rahman et al., 2012). Maiores espaçamentos permitem a oportunidade de obter plantas bem desenvolvidas, melhor enraizamento e crescimento equilibrado de ramas, de forma a promover maior absorção de nutrientes e água, o suficiente para sintetizar e assimilar fotossintéticamente, permitindo a produção de frutos maiores e de melhor qualidade. Em geral, espaçamentos mais próximos entre linhas elevam a produção de frutos devido ao aumento do número de frutos por área, mas com menor massa de fruto (Reiners \& Riggs, 1999; Fanadzo et al., 2010; El-Hamed \& Elwan, 2011; Abdel-Rahman et al., 2012).

São raros os trabalhos realizados no Brasil, onde são utilizados espaçamentos que variam em função da espécie, cultivar e sistema de produção adotado. As abóboras que apresentam plantas muito vigorosas e de hastes longas são plantadas em espaçamento de $5 \times 4$ ou $4 \times 4 \mathrm{~m}$, com duas plantas por cova. Aquelas de porte médio e os jerimuns caboclo são plantados em espaçamento de $4 \times 3 \mathrm{~m}$, com duas plantas por cova (Pedrosa et. al., 1982). Observações práticas indicam excelentes produtividades quando se cultiva apenas uma planta por cova, com aumento do número de covas por linha de plantio. Assim, espaçamentos como 4x1 ou 3x1 m, com uma planta por cova, têm proporcionado aumento do número de frutos sem prejuízo da massa do fruto (Pedrosa, 1992). Estudando diferentes espaçamentos $(3,6 \times 3,6 ; 3,0 \times 4,0 ; 3,0 \times 3,0$ e 3,0x2,0 m) com poda e sem poda da haste principal e com espaçamentos de $3,0 \times 2,0$ e 2,0x2,0 m com 1 a 4 plantas/ cova, Cortes \& Hernandez (1996) verificaram em ambos experimentos que as produtividades mais altas foram registradas para o espaçamento $3,0 \times 2,0 \mathrm{~m}$. Não foram observados efeitos da poda das plantas, no entanto, a produtividade foi influenciada significativamente pelo número de plantas/cova, que aumentou com o maior número de plantas.

A avaliação de genótipos de abóbora é ferramenta importante para a recomendação de plantio da cultura, uma vez que diferentes materiais podem responder diferentemente quanto à produtividade.

O objetivo do estudo foi avaliar acessos e cultivar de abóbora em espaçamentos entre plantas visando a sustentabilidade da produção e competividade da cultura, em áreas irrigadas, nas condições do semiárido do Vale do São Francisco.

\section{MATERIAL E MÉTODOS}

O experimento foi conduzido no período de maio a setembro de 2010 , em campo experimental da Embrapa Semiárido, em Petrolina-PE (0909'S, $40^{\circ} 22^{\prime} \mathrm{O}, 365,5 \mathrm{~m}$ de altitude). O solo classificado como ARGISSOLO VERMELHO - AMARELO Eutrófico Plíntico (Santos et al., 2006) apresentou as seguintes características químicas: $\mathrm{pH}$ $\left(\mathrm{H}_{2} \mathrm{O}\right)=6,0 ; \mathrm{Ca}^{2+}=2,1 \mathrm{cmol} \mathrm{dm}^{-3} ; \mathrm{Mg}^{2+}=$ $0,9 \mathrm{cmol}_{\mathrm{c}} \mathrm{dm}^{-3} ; \mathrm{Na}^{+}=0,01 \mathrm{cmol}_{\mathrm{c}} \mathrm{dm}^{-3}$; $\mathrm{K}^{+}=0,45 \mathrm{cmol} \mathrm{dm}^{-3} ; \mathrm{Al}^{3}=0,05 \mathrm{cmol}_{\mathrm{c}}$ $\mathrm{dm}^{-3}, \mathrm{P}($ Mehlich $)=6,00 \mathrm{mg} \mathrm{dm}^{-3} \mathrm{e} \mathrm{MO}=$ $6,1 \mathrm{~g} \mathrm{~kg}^{-1}$; as características físicas (\%) foram: areia $=82 ;$ silte $=11$ e argila $=7$. As condições climáticas durante a realização do experimento caracterizaram-se por temperatura média de $23,9^{\circ} \mathrm{C}$ com mínima de $18,1^{\circ} \mathrm{C}$ e máxima de $29,6^{\circ} \mathrm{C}$, umidade relativa média de $62,9 \%$ e precipitação acumulada no período de $27,8 \mathrm{~mm}$.

O delineamento experimental utilizado foi de blocos ao acaso, em esquema fatorial $5 \times 3$, compreendendo quatro acessos do Banco Ativo de Abóbora, selecionados pelo seu alto teor de carotenóides (A422, A612, A620 e A627) e a cultivar Jacarezinho como testemunha (bem aceita no mercado local, com características de formato de frutos desejáveis), e três espaçamentos entre plantas ( 1,2 e $3 \mathrm{~m}$ ), com quatro repetições. $\mathrm{O}$ espaçamento entre linhas foi de $4 \mathrm{~m}$. As parcelas experimentais constaram de duas linhas de $12,0 \mathrm{~m}$, as quais constituíram a área útil, retirando-se uma planta em cada extremidade, sendo utilizado como demais bordaduras linhas laterais a unidade experimental.

O solo foi preparado por meio de aração e gradagem. A adubação foi feita com a aplicação do formulado 6-2412 na dose $200 \mathrm{~kg} \mathrm{ha}^{-1}$, incorporados no sulco de plantio, sendo realizadas duas coberturas aos 20 e 45 dias após o transplante, com $50 \mathrm{~kg} \mathrm{ha}^{-1}$ de $\mathrm{Ne} 60 \mathrm{~kg}$ $\mathrm{ha}^{-1}$ de $\mathrm{k}_{2} \mathrm{O}$, usando como fontes ureia e cloreto de potássio, respectivamente.

As mudas foram obtidas em bandejas multicelulares de 128 células cada uma, preenchidas com substrato comercial (Plantmax). O transplante foi realizado aos 20 dias após o semeio em 26 de maio de 2010, quando as plantas apresentaram dois pares de folhas definitivas.

A área de cultivo foi mantida limpa, com controle de plantas invasoras realizada por meio de capinas manuais. A irrigação (aspersão) foi realizada três 
vezes por semana, com lâminas em torno de $10 \mathrm{~mm}$ baseadas na evaporação do tanque classe A e os tratos fitossanitários comuns à cultura.

A colheita foi realizada aos 16 de setembro de 2010, quando as ramas e folhas apresentavam amarelecimento, pedúnculo secos e casca resistente à penetração da unha, indicativo do ponto de colheita (Filgueira, 2008), sendo avaliadas a produtividade $\left(\mathrm{t} \mathrm{ha}^{-1}\right)$, massa fresca do fruto $\left(\mathrm{kg}_{\text {fruto }}{ }^{-1}\right)$ e número de frutos por planta e por hectare. Os dados obtidos foram submetidos à análise de variância para cada característica avaliada, aplicando-se o teste de Tukey em nível de 5\% de probabilidade para comparação das médias, empregando-se o programa SISVAR 5.0 (Ferreira, 2010).

\section{RESULTADOS E DISCUSSÃO}

Para produtividade os fatores apresentaram efeitos independentes (Tabela 1). Maior produtividade foi obtida pelo acesso A612 (17,3 $\left.\mathrm{t} \mathrm{ha}^{-1}\right)$, seguida pelo acesso A422 (15,2 $\left.\mathrm{t} \mathrm{ha}^{-1}\right)$, sendo o pior desempenho apresentado pela cultivar Jacarezinho que, sem diferenciar dos demais acessos, apresentou 12,2 $\mathrm{t} \mathrm{ha}^{-1}$. Utilizando o espaçamento de 2,5×2,5 $\mathrm{m}$ em diferentes acessos e cultivares de abóbora, Krause et al. (2006) verificaram para a cultivar Jacarezinho produtividade de 7,1 t ha-1 ${ }^{-1}$ enquanto Silva (2010), avaliando seis genótipos de abóbora no espaçamento de $3 \times 3 \mathrm{~m}$ obteve produtividades variando de 2,8 a $16,7 \mathrm{t}$ $\mathrm{ha}^{-1}$. Os resultados obtidos no presente trabalho são superiores à produtividade média nacional $\left(4,4 \mathrm{t} \mathrm{ha}^{-1}\right)$ e à mundial de abóbora $\left(13,4 \mathrm{tha}^{-1}\right)$. Os acessos mais produtivos obtiveram incrementos na produtividade, entre 293,2 e 245,4\% superiores à média nacional. Para espaçamento entre plantas observou-se que as maiores produtividades foram encontradas no menor espaçamento entre plantas (4x1 m), 18,2 t ha-1, com menor produtividade no espaçamento de 4x3 m (11,9 t ha-1). Abdel-Rahman et al. (2012), avaliando os espaçamentos 0,50; 0,75 e $1,00 \mathrm{~m}$ entre plantas, também concluíram que maiores densidades promovem melhores respostas em termos de produtividade, todavia com menor massa fresca do fruto, resultados estes corroborados por Lang \& Ermini (2010) e El-Hamed \& Elwan (2011).

Efeitos significativos independentes dos fatores estudados também foram observados para massa fresca de frutos (Tabela 1). O acesso A612 sobressaiu-se com maior massa fresca com $3,5 \mathrm{~kg}$ fruto $^{-1}$, que não diferenciou estatisticamente do acesso A422 (3,2 kg fruto $\left.{ }^{-1}\right)$. A cultivar Jacarezinho e o acesso A620 com respectivos 1,9 e 2,0 kg fruto $^{-1}$ obtiveram os menores valores. Quanto ao espaçamento, o maior $(4 \times 3 \mathrm{~m})$, destacou-se com 2,91 kg fruto ${ }^{-1}$, seguido pelos demais espaçamentos sem evidenciar diferenças significativas entre si. Utilizando o espaçamento de 2,5x2,5 $\mathrm{m}$ em diferentes acessos e cultivares de abóbora, Krause et al. (2006) verificaram valores inferiores de massa fresca, com média de $2,2 \mathrm{~kg}$ fruto $^{-1}$, obtendo para a cultivar Jacarezinho 1,7 kg fruto ${ }^{-1}$. Estes resultados são ainda comparáveis aos obtidos por Lang \& Ermini (2010) e Cushman et al. (2004) que relataram maior massa fresca de frutos na densidade mais baixa de plantio, como também Nerson (2005) relata que maiores massas de frutos foram obtidas em maiores espaçamentos, em abobrinha (Cucurbita реро). A comercialização de frutos para consumo relaciona-se à massa fresca (tamanho e peso do fruto), característica essencial de qualidade para o mercado consumidor. Nesse quesito, Ramos et al. (2010) encontraram valores entre 5 a 8 $\mathrm{kg}$ em frutos comercializados no interior da Bahia, para grupos denominados de abóbora "Maranhão" (Cucurbita moschata), e 2 a $3 \mathrm{~kg}$ para a cultivar Jacarezinho pertencente à mesma espécie. Há uma prevalência de consumo por abóboras com massa fresca de até $3 \mathrm{~kg}$. Pelo exposto, evidencia-se a importância de se estudar e/ou definir as melhores densidades de plantio em função das cultivares/acessos, que proporcionem maior ou menor massa fresca de fruto, em função das exigências do mercado. Resende \& Costa (2003) corroboram esta afirmação de ser a densidade de plantio uma ferramenta de grande importância para os agricultores, visto que, em função da demanda do mercado consumidor, pode-se manejar a densidade de plantas, visando maior retorno econômico e maximizando a produção.

Observou-se valores superiores de número de frutos por planta (Tabela 1) para a cultivar Jacarezinho (4,7 frutos planta $^{-1}$ ) e acesso A620 (4,6 frutos planta $\left.^{-1}\right)$, seguida pelos demais acessos com valores pouco inferiores, oscilando ente 3,5 e 3,8 frutos planta $^{-1}$, o que demonstrou para estes dois tratamentos alta prolificidade, no entanto, com massa fresca de fruto bem inferior. Krause et al. (2006) verificaram menor número

Tabela 1. Produtividade, massa fresca e número de frutos por planta de acessos/cultivar de abóbora, em diferentes espaçamentos (yield, fresh mass of fruits and number of fruits per plant of accessions and one cultivar of pumpkin at different spacing). Petrolina, Embrapa Semiárido, 2010.

\begin{tabular}{lccc}
\hline Acessos/cultivar & $\begin{array}{c}\text { Produtividade } \\
(\mathbf{t ~ h a} /)\end{array}$ & $\begin{array}{c}\text { Massa fresca } \\
\text { (g/fruto) }\end{array}$ & Frutos/planta \\
\hline A612 & $17,3 \mathrm{a}$ & $3,5 \mathrm{a}$ & $3,6 \mathrm{~b}$ \\
A620 & $12,8 \mathrm{c}$ & $2,0 \mathrm{c}$ & $4,6 \mathrm{a}$ \\
A422 & $15,2 \mathrm{~b}$ & $3,2 \mathrm{a}$ & $3,5 \mathrm{~b}$ \\
A627 & $13,0 \mathrm{c}$ & $2,6 \mathrm{~b}$ & $3,8 \mathrm{~b}$ \\
Jacarezinho & $12,2 \mathrm{c}$ & $1,9 \mathrm{c}$ & $4,7 \mathrm{a}$ \\
\hline Espaçamentos entre plantas $(\mathbf{m})$ & & & \\
\hline 4x1 & $18,2 \mathrm{a}$ & $2,48 \mathrm{~b}$ & $3,1 \mathrm{c}$ \\
$4 \times 2$ & $12,2 \mathrm{~b}$ & $2,49 \mathrm{~b}$ & $4,1 \mathrm{~b}$ \\
4x3 & $11,9 \mathrm{~b}$ & $2,91 \mathrm{a}$ & $5,0 \mathrm{a}$ \\
\hline CV (\%) & 7,4 & 10,3 & 11,0 \\
\hline
\end{tabular}

*Médias seguidas da mesma letra nas colunas não diferem entre si pelo teste de Tukey a $5 \%$ de probabilidade (means followed by the same lowercase letter in column do not differ by Tukey test at $5 \%$ probability). 
Tabela 2. Número de frutos por hectare de acessos/cultivar de abóbora em diferentes espaçamentos (number of fruits per hectare of accessesions and one cultivar of pumkin at different spacing). Petrolina, Embrapa Semiárido, 2010.

\begin{tabular}{lcccc}
\hline \multirow{2}{*}{ Acessos/cultivar } & \multicolumn{3}{c}{ Espaçamentos (m) } & \multirow{2}{*}{ Médias } \\
\cline { 2 - 4 } & $\mathbf{4 x \mathbf { 1 }}$ & $\mathbf{4 x 2}$ & $\mathbf{4 x 3}$ & \\
\hline A612 & $6528 \mathrm{a} \mathrm{C}$ & $4583 \mathrm{~b} \mathrm{~B}$ & $3764 \mathrm{~b} \mathrm{AB}$ & $4958 \mathrm{~B}$ \\
$\mathrm{~A} 620$ & $8125 \mathrm{a} \mathrm{B}$ & $6319 \mathrm{~b} \mathrm{~A}$ & $4722 \mathrm{c} \mathrm{AB}$ & $6389 \mathrm{~A}$ \\
A422 & $6528 \mathrm{a} \mathrm{C}$ & $4514 \mathrm{~b} \mathrm{~B}$ & $3542 \mathrm{~b} \mathrm{~B}$ & $4861 \mathrm{~B}$ \\
A627 & $7222 \mathrm{a} \mathrm{BC}$ & $4653 \mathrm{~b} \mathrm{~B}$ & $3889 \mathrm{~b} \mathrm{AB}$ & $5255 \mathrm{~B}$ \\
Jacarezinho & $9722 \mathrm{a} \mathrm{A}$ & $5417 \mathrm{~b} \mathrm{AB}$ & $4861 \mathrm{~b} \mathrm{~A}$ & $6667 \mathrm{~A}$ \\
\hline Médias & $7625 \mathrm{a}$ & $5097 \mathrm{~b}$ & $4155 \mathrm{c}$ & \\
CV $(\%)=9,1$ & & & & \\
\hline
\end{tabular}

*Médias seguidas da mesma letra nas colunas não diferem entre si pelo teste de Tukey a 5\% de probabilidade (means followed by the same lowercase letter in column do not differ by Tukey test at $5 \%$ probability).

médio de frutos por planta (2,6 frutos planta $^{-1}$ ) em diferentes linhagens e cultivares de abóbora no espaçamento de $2,5 \times 2,5 \mathrm{~m}$. O aumento da densidade de plantas diminuiu o tamanho dos frutos e o número de frutos por planta (Lang \& Ermini, 2010).

Com relação a número de frutos por hectare os resultados demonstraram efeitos da interação (Tabela 2). Pelo desdobramento de espaçamentos dentro de acessos constataram-se para todos os acessos que maior número de frutos por hectare foram obtidos no espaçamento de $4 \times 1 \mathrm{~m}$, seguido pelos espaçamentos de 4x2 e 4x3 m, que não estabeleceram diferenças significativas entre si, à exceção do acesso A620 que registrou diferenças entre os três espaçamentos avaliados. Desdobrando-se a interação no sentido de acesso dentro de espaçamento, detecta-se, de forma geral, que o acesso A620 e Jacarezinho apresentaram maior número de frutos por hectare, ocorrendo para os demais acessos valores comparáveis, o que pode ser observado pelas médias de número de frutos registrados. Resultados semelhantes foram obtidos por Lang \& Ermini (2010) que verificaram que o aumento do espaçamento contribuiu para produção de menor número de frutos por área.

Maiores espaçamentos permitem obter plantas bem desenvolvidas, melhor enraizamento e crescimento equilibrado de ramas, de forma a promover maior absorção de nutrientes e água, o suficiente para sintetizar e assimilar fotos- sinteticamente, permitindo a produção de frutos maiores e com qualidade. Em geral, espaçamentos mais próximos entre linhas elevam a produtividade de frutos por meio de aumento do número de frutos, mas com menor massa fresca de fruto (Reiners \& Riggs, 1999; White, 2001; Cushman et al., 2004; Fanadzo et al., 2010; El-Hamed \& Elwan, 2011; Abdel-Rahman et al., 2012). Esse fato, segundo Robinson \& Walters (1997), tem sido atribuído principalmente às pressões de competição inter e intraespecífica.

Em função dos resultados obtidos no presente trabalho, pode-se recomendar o plantio dos acessos A612 e A422 como os mais produtivos. Em termos de espaçamento entre plantas, o de $4 \times 1 \mathrm{~m}$ é o mais indicado; no entanto, se a preferência do mercado consumidor recair sobre frutos de maior massa fresca (tamanho), sugere-se o espaçamento de $4 \times 3 \mathrm{~m}$.

\section{AGRADECIMENTOS}

A autora Nadja Pollyanna S Gonçalves agradece à FACEPE pela concessão de bolsa.

\section{REFERÊNCIAS}

ABDEL-RAHMAN MSS; EL-DKESHY MHZ; ATTALLAH SY. 2012. Plant spacing with seed chilling or plant girdling affect of Pumpkin (C. moschata) growth and yield components. Research Journal of Agriculture and Biological Sciences 8: 6-10.

AMERI A, NASSIRI M; REZVANI P. 2007.
Effects of different nitrogen levels and plant density on flower, essential oils and extract production and nitrogen use efficiency of Marigold (Calendula officinalis). Iranian Journal Field Crops Research 5: 315-325.

BARBIERI RL; HEIDEN G; NEITZKE RS; GARRASTAZÚ MC; SCHWENGBER JE. 2006. Banco Ativo de Germoplasma de Cucurbitáceas da Embrapa Clima Temperado - periodo de 2002 a 2006. Pelotas: Embrapa Clima Temperado. 30p. (Documento, 176).

BORGES RME; RESENDE GM; LIMA MAC; DIAS RCS; LUBARINO PCC; OLIVEIRA RCS; GONÇALVES NPS. 2011. Phenotypic variability among pumpkin accessions in the Brazilian semiarid. Horticultura Brasileira 29: 461-464.

CARMO GA; OLIVEIRA FRA; MEDEIROS JF; OLIVEIRA FA; CAMPOS MS; FREITAS DC. 2011. Teores foliares, acúmulo e partição de macronutrientes na cultura da abóbora irrigada com água salina. Revista Brasileira de Engenharia Agrícola e Ambiental 15: 512-518.

CORTES S; HERNANDEZ A. 1996. La densidad de siembra en calabaza (Cucurbita moschata Duch) variedad Mariucha en dos fechas de siembra y su influencia en los rendimientos. Cultivos Tropicales 17: 44-47.

CUSHMAN KE; NAGEL DH; HORGAN TE; GERARD PD. 2004. Plant population affects pumpkin yield components. HortTechnology 14: 326-331.

EL-HAMED KEA; ELWAN MWM. 2011. Dependence of pumpkin yield on plant density and variety. American Journal of Plant Sciences 2:636-643.

FANADZO M; CHIDUZA C; MNKENI PNS. 2010. Pre-plant weed control, optimum N rate and plant densities increase butternut (Cucurbita moschata) yield under smallholder irrigated conditions in the Eastern Cape Province of South Africa. African Journal of Agricultural Research 16: 2192-2199.

FAO. Agricultural production, primary crops. 2012. Disponível em: <http://www.fao.org> Acesso em: 16 jun. 2012.

FERREIRADF. 2010. SISVAR Versão 5.3. Lavras: Departamento de Ciências Exatas, UFLA.

FERREIRA MAJ; MELO AMT; CARMO CAS; SILVA DJH; LOPES JF; QUEIROZ MA; MOURA MCCL; DIAS, RCS; BARBIERI RL; BARROZO LV; GONÇALVES EM; NEGRINI ACA. 2006. Mapeamento da distribuição geográfica e conservação dos parentes silvestres e variedades crioulas de Cucurbita. In: Parentes Silvestres das espécies de plantas cultivadas. Secretaria de Biodiversidade e Florestas. Brasília. 44p.

FILGUEIRA FAR. 2008. Novo manual de olericultura: agrotecnologia moderna na produção e comercialização de hortaliças. Viçosa: Editora UFV, 3 ed. 421p.

HEIDEN G; BARBIERI RL; NEITZKE RS. 2007. Chaves para a identificação das espécies de abóbora (Cucurbita, Cucurbitaceae) cultivadas no Brasil. Pelotas: Embrapa Clima Temperado. 31p. (Documentos, 197).

INSTITUTO BRASILEIRO DE GEOGRAFIA E ESTATÍsTICA (IBGE). 2012. Produção Agrícola Municipal. Disponível em <www. 
sidra.ibge.gov.br.> Acesso em 16 fev. 2012.

INSTITUTO BRASILEIRO DE GEOGRAFIA E ESTATÍSTICA (IBGE). 2010. Pesquisa de orçamento familiares: 2008-2009. Rio de Janeiro: IBGE, 282p.

KRAUSE W; BEZERRA NETO FV; LEAL NR; GONÇALVES MG; MORENZ EF. 2006. Produção e características de frutos de abóbora em Seropédica-RJ. In: Congresso Brasileiro de Olericultura, 46. Goiânia. Anais... Goiânia: UFG.

LATIFI M ; BARIMAVANDI A ; SEDAGHATHOOR S; LIPAYI SR. 2012. Sowing date and plant population effects on seed yield of Cucurbita pepo. International Journal Agriculture Biology 14: 641-644.

LANG MY; ERMINI P. 2010. Evaluación de distintas densidades de siembra en un cultivo de zapallo tipo "Anco" (Cucurbita moschata) en la región semiárida Pampeana. Revista de la Facultad de Agronomía de la Universidad Nacional de La Pampa 21: 39-45.
NERSON H. 2005. Effects of fruit shape and plant density on seed yield and quality of squash. Scientia Horticulturae 105: 293-304.

PEDROSA JF. 1992. Cultura dos jerimuns. Mossoró: ESAM, 31 p. (apostila).

PEDROSA JF; ALVARENGA MR; FERREIRA FA; CASALI VWD. 1982. Abóboras, morangas e abobrinhas: cultivares e métodos culturais. Informe Agropecuário 8: 24-26.

RAMOS SSR; LIMA NRS; CARVALHO HWL; OLIVEIRA IR; SOBRAL FS; CURADO FF. 2010. Aspectos técnicos do cultivo da abóbora na região Nordeste do Brasil. Aracaju: Embrapa Tabuleiros Costeiros, 36p. (Embrapa Tabuleiros Costeiros. Documentos, 154).

REINERS S; RIGGS DIM. 1999. Plant population affects yield and fruit size of pumpkin. HortScience 34: 1076-1078.

RESENDE GM; COSTAND. 2003. Características produtivas da melancia em diferentes espaçamentos de plantio. Horticultura Brasileira 21: 697-700.
ROBINSON RW; WALTERS DSD. 1997. Cucurbits. New York: CAB International, 226p.

SANTOS HG; JACOMINE PKT; ANJOS LHC; OLIVEIRA VA; OLIVEIRA JB; COELHO MR; LUMBRERAS JF; CUNHA TJF. (ed). 2006. Sistema brasileiro de classificação de solos. 2. ed. Rio de Janeiro: Embrapa Solos. $306 \mathrm{p}$.

SILVA TB. 2010. Seleção, comportamento fenotipico e genotipico e desenvolvimento de uma nova cultivar de abóbora (Cucurbita moschata Dusch). Sergipe: Universidade Federal de Sergipe. 34p (Dissertação mestrado).

TEPPNER H. 2004. Notes on Lagenaria and Cucurbita (Cucurbitaceae). Phyton 44: 245 308.

WHITE JM. 2001. Calabaza yield and size at two spacings when grown on various plastic mulches as a second crop. Proceedings Florida State Horticultural Society 144: 335-336. 